\title{
Notch 2 signaling contributes to cell growth, anti-apoptosis and metastasis in laryngeal squamous cell carcinoma
}

\author{
YOU ZOU $^{1 *}$, FANG FANG $^{2 *}$, YONG-JUN DING $^{1}$, MENG-YUAN DAI $^{1}$, XING YI $^{1}$, \\ CHEN CHEN $^{1}$, ZE-ZHANG TAO ${ }^{1,3}$ and SHI-MING CHEN ${ }^{1,3}$
}

\begin{abstract}
Departments of ${ }^{1}$ Otolaryngology Head and Neck Surgery and ${ }^{2}$ Medical Market, Renmin Hospital of Wuhan University; ${ }^{3}$ Otolaryngology Head and Neck Surgery Institute, Medical School of Wuhan University, Wuhan, Hubei 430060, P.R. China
\end{abstract}

Received June 18, 2015; Accepted May 31, 2016

DOI: $10.3892 / \mathrm{mmr} .2016 .5688$

\begin{abstract}
Notch signaling is important during the development of a variety of human tumors. Depending on the context, Notch signaling can be either oncogenic or anti-proliferative, and therefore, its effects in cancer are unpredictable. The aim of the present study was to identify the importance of Notch 2 in the cell growth and metastasis of laryngeal squamous cell carcinoma (LSCC). The current study performed quantum dots-based immunofluorescence histochemistry to determine expression of Notch 2 in 72 LSCC samples without lymph node metastasis, 23 LSCC samples with lymph node metastasis and 31 samples from vocal cord polyps. It was observed that Notch 2 was upregulated in LSCC tissue compared with normal vocal cord polyps. This upregulation was further enhanced in LSCC tissues with lymph node metastasis compared with LSCC tissues without lymph node metastasis. Following knockdown of NOTCH2 expression in LSCC cells, the in vitro tumorigenicity of Hep-2 cells was inhibited, with growth, migration, invasion and proliferation reduced, and apoptosis induced. Additionally, following downregulation of Notch 2 protein expression, the protein expression levels of phospho-mitogen-activated protein kinase 1 (p-ERK), $v$-myc avian myelocytomatosis viral oncogene homolog and B-cell CLL/lymphoma 2 ( $\mathrm{Bcl} 2)$ were also downregulated, whereas, $\mathrm{Bcl} 2$-associated $\mathrm{X}$ protein expression was upregulated. There were no changes detected in the protein expression levels of total-ERK, phospho-v-akt murine thymoma viral oncogene homolog 1 (p-Akt) and total-Akt. The results of the present study suggest that Notch 2 is important for the cell growth, anti-apoptosis and metastasis of LSCC. Therefore, Notch 2
\end{abstract}

Correspondence to: Professor Shi-Ming Chen, Department of Otolaryngology Head and Neck Surgery, Renmin Hospital of Wuhan University, 238 Jie Fang Road, Wuhan, Hubei 430060, P.R. China E-mail: shimingchen0468@163.com

*Contributed equally

Key words: Notch 2, laryngeal squamous cell carcinoma, apoptosis, proliferation, metastasis inhibitors may have therapeutic potential for the treatment of patients with LSCC via the inhibition of cancer cell growth and metastasis.

\section{Introduction}

One of the common types of malignant cancer affecting the head and neck is laryngeal cancer. As these types of cancer are largely derived from the skin of the larynx, the majority are cases of laryngeal squamous cell carcinoma (LSCC). While early-stage LSCC is often curable with surgery or radiotherapy, patients with advanced carcinoma have poor long-term survival rates $(1,2)$, as LSCCs are often difficult to remove completely, and cancer recurrence following treatment remains a significant obstacle.

Despite advances in anticancer therapeutics, the outcome for advanced LSCC patients has not improved in the last two decades. This is due to the fact that the molecular mechanisms involved in the initiation and progression of LSCC remain largely unknown (3). Therefore, understanding the molecular mechanisms of LSCC progression is crucial for improving therapies and the long-term prognosis of patients.

One pathway that has been implicated in cell survival, angiogenesis and resistance to therapy in various different tumors is the Notch signaling pathway (4). Notch signaling is crucial during embryonic development and in adult life. For the pathway to be activated, one of the four Notch receptors expressed in mammals (Notch 1-4) will interact with one of the five known Notch ligands, jagged 1, jagged 2, Delta-like (DLL)1, DLL3 or DLL4, present on an adjacent cell. Following ligand binding, the intracellular domain of Notch (NICD) is cleaved by metalloproteases and $\gamma$-secretases. The NICD then translocates from the plasma membrane to the nucleus, where it can drive the expression of numerous genes, including hes-related family bHLH transcription factor with YRPW motif 1 , hes family bHLH transcription factor $1, v$-myc avian myelocytomatosis viral oncogene homolog (Myc), cyclin D1 and B-cell CLL/lymphoma 2 (Bcl-2), which regulate multiple cellular processes, including cell proliferation, differentiation, stem cell maintenance and apoptosis.

Notch pathway signaling has been previously reported be tumor-suppressive and oncogenic $(5,6)$. Aberrant activation of this pathway has been associated with tumorigenesis in 
prostate cancer development and metastasis $(7,8)$. Similarly, expression of the Notch ligand jagged 1 is correlated with increased aggressiveness of gastric cancer and poor patient survival rates (9). Dysregulation of Notch signaling has been previously reported in human hematological malignancies and in various solid tumors (6), including cases of T-cell (10-12) and B-cell (13-15) carcinoma. Additionally, Notch signaling maintains the survival of stem and precursor cells in multiple tissues, including the gut and glandular tissues, and thus, also maintains the survival of cancer stem cells (16).

Notch signaling has also been previously demonstrated to promote tissue differentiation, notably, in the squamous epithelia of various organs and the skin. Therefore, mutations that inactivate crucial components of the Notch signaling pathway, including mutations in the Notch 1, 2 or 3 receptors, may result in squamous cell carcinomas, including LSCC. However, the precise mechanisms of the Notch pathway in LSCC development remain unclear.

Multiple studies have reported that the Notch 2 signaling pathway in particular has important potential oncogenic or tumor-suppressing actions in several hematologic malignancies, including multiple myeloma, B cell chronic lymphocytic leukemia, B and T cell acute lymphoblastic leukemia, and also in various solid tumors, including glioblastoma and breast, cervical, colon, pancreatic, skin and small cell lung cancer $(17,18)$. Consequently, the current study analyzed the expression of Notch 2 in clinical LSCC samples using quantum dots (QDs)-based immunofluorescence histochemical staining. Additionally, knockdown of NOTCH2 was performed in a Hep-2 cell line using short hairpin RNA (shRNA). These studies aimed to elucidate the effects of Notch 2 on the proliferation, migration and invasion of LSCC.

\section{Materials and methods}

Patients. In total, 95 laryngeal carcinoma samples with adjacent non-tumor tissues were obtained from patients who had undergone surgery between 2009 and 2013 at the Renmin Hospital of Wuhan University (Wuhan, China). All paraffin-embedded tissue specimens were analyzed and reconfirmed by two experienced pathologists. The clinicopathologic characteristics of the patients were squamous cell carcinoma. All patients underwent selective neck lymph node dissection and lymph node tissues were analyzed by pathologists. If the clinicopathologic characteristics of lymph nodes tissue were positive, the tissue was confirmed as LSCC with lymph node metastasis. If the clinicopathologic characteristics of the lymph node tissue was negative, the tissue of patients was confirmed as LSCC without lymph node metastasis. The present study was approved by the Ethics Committee of Renmin Hospital of Wuhan University, and written informed consent was obtained from each participant.

Laryngeal carcinoma specimens and cell lines. The Hep-2 cell line was obtained from the China Center for Type Culture Collection (Wuhan, China). Hep-2 cells were cultured in RPMI-1640 (HyClone; GE Healthcare Life Sciences, Logan, UT, USA) with $10 \%$ fetal bovine serum (FBS; Gibco; Thermo Fisher Scientific, Inc., Waltham, MA, USA) and incubated in a humidified atmosphere with $5 \% \mathrm{CO}_{2}$ at $37^{\circ} \mathrm{C}$.
RNA interference of NOTCH2 using shRNA. The enhanced green fluorescent protein (EGFP)-V-RS-Notch 2 shRNA plasmids were synthesized by Wuhan XiMa Technologies Co., Ltd. (Wuhan, China). Cells were transfected with shRNA vectors using Lipofectamine RNAiMAX (Invitrogen; Thermo Fisher Scientific, Inc.), according to the manufacturer's instructions. The structure of the shRNA plasmid was as follows: Stop-mir30-flanking-shRNA1-mir30-flanking-EG FP-CMV-U6-shRNA2-stop. The shRNA sequences were as follows: Notch2 shRNA1, 5'-TGGAGGTCTCAGTGGATA TAA-3'; and Notch2 shRNA2, 5'-AAGATCCTGTTAGAC CATTT-3'. Three treatments were designed for the current study. Untreated Hep-2 cells were considered the blank control and termed the non-transfected group. The cells transfected with the EGFP-V-RS-negative shRNA plasmid, containing non-specific shRNA, and the EGFP-V-RS-Notch 2 shRNA plasmid, containing the Notch 2-specific shRNA, were termed the negative-shRNA and Notch 2-shRNA groups, respectively.

QDs-based immunofluorescence histochemistry. Tissue sections were deparaffinized in xylene and rehydrated in a graded ethanol series. For antibody binding, slides were first incubated with $2 \%$ bovine serum albumin (BSA; Gibco Thermo Fisher Scientific, Inc.) at $37^{\circ} \mathrm{C}$ for $30 \mathrm{~min}$, and then incubated with primary rabbit anti-prostate stem cell antigen antibody (rabbit polyclonal anti-Notch2 antibody; cat. no. SAB4502020, Sigma-Aldrich, St. Louis, MO, USA, 1:100) diluted in Tris-buffered saline (TBS) overnight at $4^{\circ} \mathrm{C}$. Slides were then washed in TBS. Negative control samples were prepared in parallel, in which the primary antibody was replaced by TBS.

For QD conjugation, slides were incubated with 2\% BSA at $37^{\circ} \mathrm{C}$ for $10 \mathrm{~min}$, and then incubated with $\mathrm{ZnS}$-capped CdSe QDs conjugated anti-rabbit IgG probes (Wuhan Jiayuan Quantum Dot Technological Development Co., Ltd., Wuhan, China), with an emission wavelength of $605 \mathrm{~nm}$, diluted $1: 50$ in $2 \% \mathrm{BSA}$ for $30 \mathrm{~min}$ at $37^{\circ} \mathrm{C}$. Following incubation, the slides were vigorously washed with TBS, mounted with neutral glycerol, and stored at $4^{\circ} \mathrm{C}$ until observation.

The QDs were excited by blue light (excitation wavelength of 450-480 nm under U-MWB filters) and the subsequent emission of red light was monitored. The immunohistochemical staining was observed under a light microscope. Positive cells expressing Notch 2 exhibited brown-yellow staining and were granular in appearance. During the observation period, all labeled slides were stored at $4^{\circ} \mathrm{C}$, primarily to prevent the drying of tissues. The stained samples were scored using the extensional standard as follows: i) Number of positively stained cells $\leq 5 \%$ scored $0,6-25 \%$ scored $1,26-50 \%$ scored 2 , $51-75 \%$ scored $3,>75 \%$ scored 4 ; and ii) intensity of stain, colorless scored 0 , pallide-flavens scored 1 , yellow scored 2 , brown scored 3 . The staining score was obtained by multiplying the number of positively stained cells and the intensity of stain, and stratified as either negative ( $<3$ score) or strong ( $\geq 3$ score) (19).

Reverse transcription-quantitative polymerase chain reaction $(R T-q P C R)$. Total RNA was extracted from cells with TRIzol reagent (Invitrogen; Thermo Fisher Scientific, Inc.) and reverse transcribed to cDNA with the PrimeScript RT 
Table I. Expression of Notch 2 in LSCC tissue and samples from vocal cord polyps.

\begin{tabular}{|c|c|c|c|c|}
\hline Sample & Total, $\mathrm{n}$ & Negative, n (\%) & Positive, n (\%) & P-value \\
\hline Vocal cord polyp & 31 & $21(67.74)$ & $10(32.26)$ & \\
\hline LSCC without lymph node metastasis & 72 & $12(16.67)$ & $60(83.33)$ & $<0.05^{\mathrm{a}}$ \\
\hline LSCC with lymph node metastasis & 23 & $0(0.00)$ & $23(100.00)$ & $<0.05^{\mathrm{a}, \mathrm{b}}$ \\
\hline
\end{tabular}

${ }^{\mathrm{a}} \mathrm{P}=0.001$ vs. vocal cord polyps. ${ }^{\mathrm{b}} \mathrm{P}=0.037$ vs. $\mathrm{LSCC}$ without lymph node metastasis. $\mathrm{LSCC}$, laryngeal squamous cell carcinoma.

Reagent kit (Takara Biotechnology Co., Ltd., Dalian, China), according to the manufacturer's protocol. The synthesized cDNA was used as the template to detect the expression of the genes of interest by RT-qPCR with SYBR Premix Ex Taq (Takara Biotechnology Co., Ltd.). Primer sequences were as follows: NOTCH2, forward 5'-ATCCCACAAAGCCTA GCACC-3', reverse 5'-CCTTGTCCCTGAGCAACCAT-3'; and GAPDH, forward 5'-GAAAGCCTGCCGGTGACTAA-3', reverse 5'-AGGAAAAGCATCACCCGGAG-3'. qPCR was conducted using LightCycler Technology (LC-96; Roche, Mannheim, Germany). Cycling conditions included reverse transcription at $50^{\circ} \mathrm{C}$ for $30 \mathrm{~min}$, denaturation at $95^{\circ} \mathrm{C}$ for $15 \mathrm{~min}, 40$ cycles of $94^{\circ} \mathrm{C}$ for $30 \mathrm{sec}, 55^{\circ} \mathrm{C}$ for $30 \mathrm{sec}$, and $68^{\circ} \mathrm{C}$ for $2 \mathrm{~min}$, and a final extension step at $68^{\circ} \mathrm{C}$ for $10 \mathrm{~min}$. PCR experiments were repeated 3 times. Data were analyzed according to the $2^{-\Delta \Delta \mathrm{Cq}}$ method (20).

Cell viability assay. Cells were transfected with shRNA for $24 \mathrm{~h}$, then plated in 96-well culture plates. At the same time each day for three consecutive days, the original culture medium was removed, and $10 \mu \mathrm{l}$ cell counting kit-8 (CCK-8) dye (Dojindo Molecular Technologies, Inc., Kumamoto, Japan) and $90 \mu \mathrm{l}$ fresh RPMI-1640 were added to each well. The cells were incubated at $37^{\circ} \mathrm{C}$ for $1 \mathrm{~h}$. Absorbance $(450 \mathrm{~nm})$ of the medium was measured with an ELx-800 plate reader (BioTek China, Beijing, China). Representative data for three independent experiments are presented.

Apoptosis assay. Cellular apoptosis was analyzed using the FACScan instrument (BD Biosciences, San Jose, CA, USA). The cells were harvested and washed in phosphate-buffered saline, then subsequently stained with annexin $\mathrm{V}$-allophycocyanin and propidium iodide 7-amino actinomycin D (BD Pharmingen, San Diego, CA, USA). Cells were quantified using a BD FACSVerse flow cytometer (BD Biosciences). Quantification of the apoptotic fraction included early and late apoptotic cells. All data were analyzed using Kaluza software 1.1 (Beckman Coulter, Brea, CA, USA). Representative data for three independent experiments are presented.

Scratch wound healing motility assay. Cells were transfected with shRNA for $24 \mathrm{~h}$ and then plated in 6-well culture plates. A 'scratch' was created by running a pipette tip through the plate. Cells were then cultured under standard conditions for a further $24 \mathrm{~h}$. Plates were washed twice with fresh medium to remove non-adherent cells and then images were captured. The number of cells that had migrated from the edge of the wound were counted. Results were expressed as the average number of cells per field. Representative data for three independent experiments are presented.

In vitro cell invasion assay. Transwell membranes (Corning Incorporated, Corning, NY, USA) were coated with Matrigel $(2.5 \mathrm{mg} / \mathrm{ml})$. Cells were transfected with shRNA for $24 \mathrm{~h}$, then serum starved for $8 \mathrm{~h}$ and collected in RPMI-1640 medium containing 3\% FBS. Cells were seeded onto the upper wells of pre-coated Transwell inserts in the same medium at a density of $1.0 \times 10^{5}$ cells/well. Lower wells of pre-coated Transwell inserts contained $800 \mu$ l RPMI-1640 supplemented with $10 \%$ FBS. After 48 h, membranes were swabbed with a Q-tip and stained with crystal violet prior to cell counting under a microscope. Representative data for three independent experiments are presented.

In vitro cell migration assay. Cell migration assays were also performed using the Transwell membranes. The procedure used for this assay was similar to that of the cell invasion assay, except that the Transwell membrane was not coated with Matrigel. Three to four fields on each filter were scored under an inverted microscope in each experiment. Representative data for three independent experiments are presented.

Western blot assay. Total protein from Hep-2 cells transfected with the plasmids was extracted using radioimmunoprecipitation assay buffer $\left(1 \mathrm{mM} \mathrm{MgCl}_{2}, 10 \mathrm{mM}\right.$ Tris- $\mathrm{HCl} \mathrm{pH} 7.4,1 \%$ Triton X-100, 0.1\% SDS, 1\% NP-40). The sample was then centrifuged at $4^{\circ} \mathrm{C}, 12,000 \mathrm{x}$ g for $15 \mathrm{~min}$. The supernatant was isolated and protein expression was analyzed by western blot analysis. The amount of protein loaded for each lane was quantified at $25 \mu \mathrm{g}$. Total protein extracts were separated on $12 \%$ SDS-PAGE gels and transferred to polyvinylidene difluoride membranes. GAPDH was used as a loading control. The membrane was blocked using $5 \%$ skimmed milk powder at $4^{\circ} \mathrm{C}$ for $60 \mathrm{~min}$. The membranes were immunoblotted with the following primary antibodies: Anti-p-ERK (1:1,000 rabbit monoclonal antibody, cat. no. 4695, Cell Signaling Technology, Danvers, MA, USA, ); anti-t-ERK (rabbit monoclonal antibody, cat. no. 5013, Cell Signaling Technology, 1:1,000); anti-p-AKT (rabbit monoclonal, cat. no. 4060, Cell Signaling Technology, 1:1,000); t-AKT (rabbit monoclonal antibody, cat. no. 4691, Cell Signaling Technology, Inc., 1:1,000); anti-c-myc (rabbit monoclonal antibody, cat. no. 13987, Cell Signaling Technology, 1:1,000); anti-Bax (rabbit monoclonal antibody, cat. no. 5023, Cell Signaling Technology, Inc., 1:1,000); anti-Bcl-2 (rabbit monoclonal antibody, cat. no. 4223, Cell 

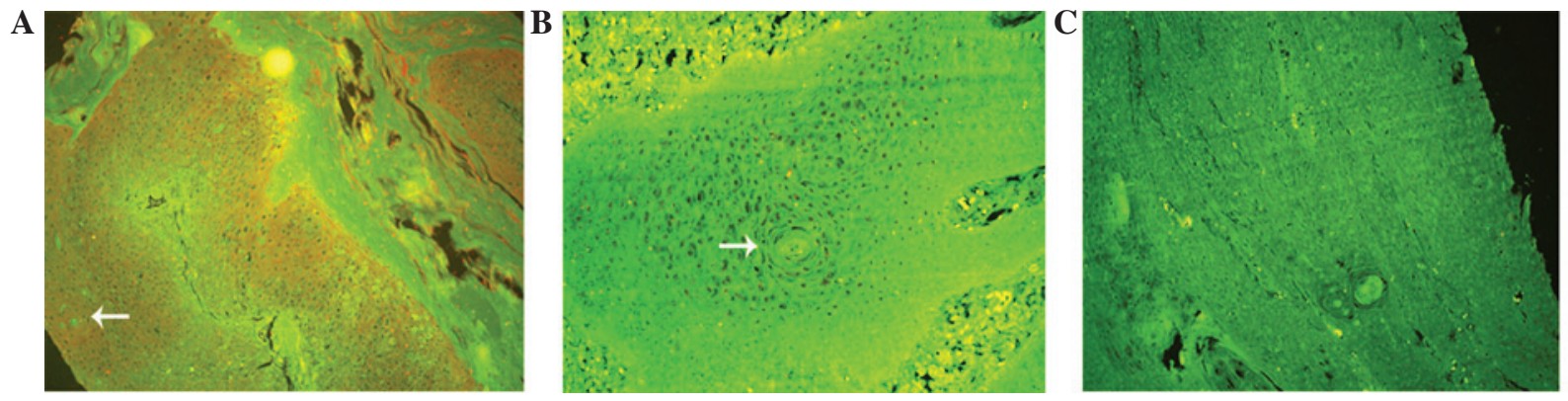

Figure 1. Notch 2 expression level is upregulated in LSCC samples. Representative images of quantum dots-based immunofluorescence histochemistry for Notch 2 expression in tissue from (A) LSCC with lymph node metastasis, (B) LSCC without lymph node metastasis and (C) vocal cord polyps. Magnification, $\mathrm{x} 100$. White arrows indicate the Notch 2 expression. LSCC, laryngeal squamous cell carcinoma.

A

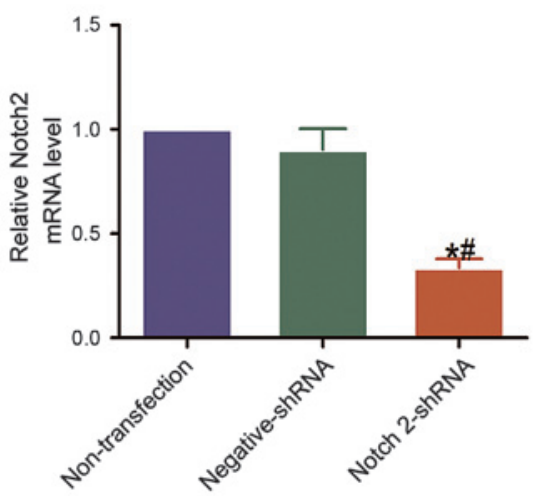

B
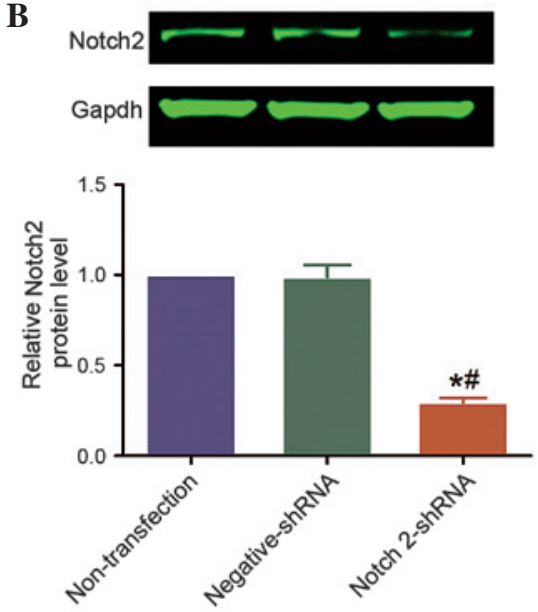

Figure 2. NOTCH2 was knocked down using shRNA. The expression of NOTCH2 mRNA and Notch 2 protein in Hep-2 cells 48 h after shRNA transfection measured by (A) reverse transcription-quantitative polymerase chain reaction, and (B) western blot with densitometric analysis, respectively. Notch 2 -shRNA samples demonstrate a significantly reduced level of Notch 2 . The data represent the results of three independent experiments. ${ }^{*}<0.05$ vs. non-transfected samples, ${ }^{\#} \mathrm{P}<0.05$ vs. negative-shRNA samples. No significant difference was observed between the non-transfected and the negative-shRNA samples $(\mathrm{P}>0.05)$. shRNA, short hairpin RNA.

Signaling Technology, Inc., 1:1,000). The membranes were then washed three times using TBS with $0.1 \%$ Tween 20 , and incubated with Donkey anti-rabbit IgG secondary antibody (cat. no. ab175731; Abcam, Cambridge, MA, USA; 1:10,000). Enhanced chemiluminescence detection (Cell Signaling Technology) was used to observe the blots. The densitometry of the bands was quantified using the Image $\mathrm{J}$ version $1.38 \mathrm{X}$ software (imagej.nih.gov/ij). Western blotting was performed in triplicate.

Statistical analysis. The statistical analysis of Notch 2 QDs-based immunofluorescence histochemistry was performed using the Wilcoxin rank-sum test. The other results were analyzed using one-way analysis of variance followed by Bonferonni's method. The statistical analyses were performed using SPSS software (version 17.0; SPSS, Inc., Chicago, IL, USA) $\mathrm{P}<0.05$ was considered to indicate a statistically significant difference.

\section{Results}

Notch 2 protein levels upregulated in LSCC. The present study examined the expression of Notch 2 in a total of 126 samples from LSCC tissues and vocal cord polyps. Among the primary LSCC tissues, 83/95 (87.4\%) cases exhibited Notch 2 expression, whereas, 10/31 (32.3\%) cases from vocal cord polyps exhibited Notch 2 expression (Table I). QDs staining demonstrated that the Notch 2 protein is located in the cytoplasm and the nucleus (Fig. 1). Notch 2 expression was absent or low in vocal cord polyps compared with LSCC samples. The protein expression rates of Notch 2 in LSCC samples with $(\mathrm{P}=0.001)$ and without $(\mathrm{P}=0.001)$ lymph node metastasis were increased compared with the levels in vocal cord polyp samples (Table I). Additionally, the expression of Notch 2 was increased in tissue samples from LSCC with lymph node metastasis compared with those without metastasis $(\mathrm{P}=0.037)$, which indicates that Notch 2 may be important in lymph node metastasis of LSCC.

Notch 2 protein expression is stably and effectively downregulated by shRNA in Hep-2 laryngeal cancer cells. The efficacy of shRNA-mediated knockdown of NOTCH2 in Hep-2 cells and the successful transfection of Notch2-shRNA plasmid transfection into Hep-2 cells were analyzed using RT-qPCR and western blotting. NOTCH2 mRNA levels were reduced by 82.0 and $80.3 \%$ in Notch2-shRNA-transfected cells compared with cells that were non-transfected or transfected with negative-shRNA, 
A
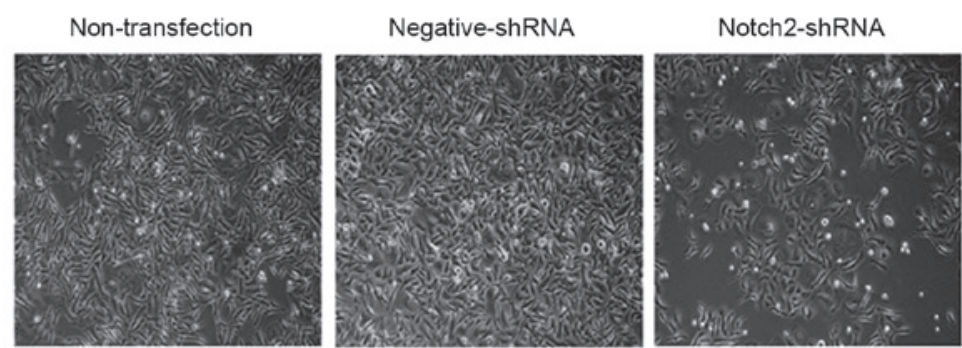

C
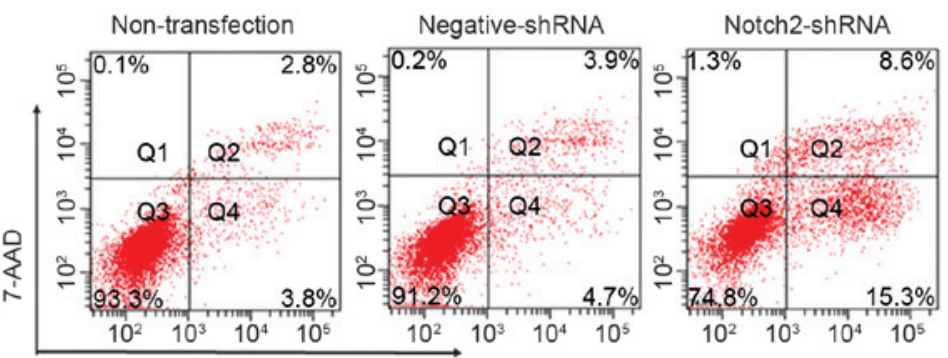

Annexin V-APC
B

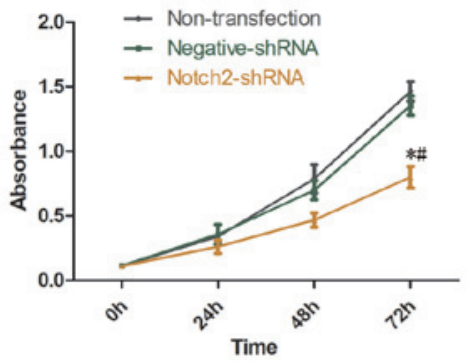

D

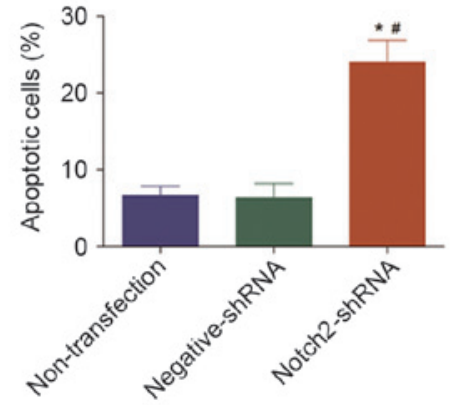

Figure 3. Effects of Notch 2 on cell morphology and viability. Notch 2-shRNA cells exhibited a higher rate of apoptosis compared with non-transfected or negative-shRNA cells. (A) Photographs of Hep-2 cell morphology captured $72 \mathrm{~h}$ after shRNA transfection. (B) Proliferation of Hep-2 cells was detected by Cell Counting kit-8 reagent following shRNA transfection. (C) Flow cytometry data of apoptotic Hep-2 cells and (D) the percentage of apoptotic Hep-2 cells $72 \mathrm{~h}$ after shRNA transfection. The data represent the results of three independent experiments. ${ }^{*} \mathrm{P}<0.05$ vs. non-transfected cells, ${ }^{"} \mathrm{P}<0.05 \mathrm{vs}$. negative shRNA. shRNA, short hairpin RNA.

A
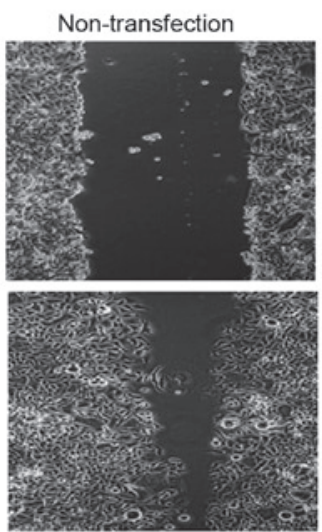

C

Non-transfection

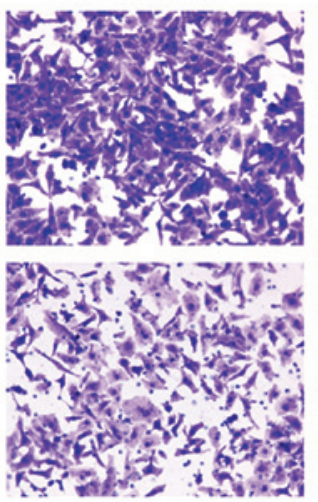

Negative-shRNA
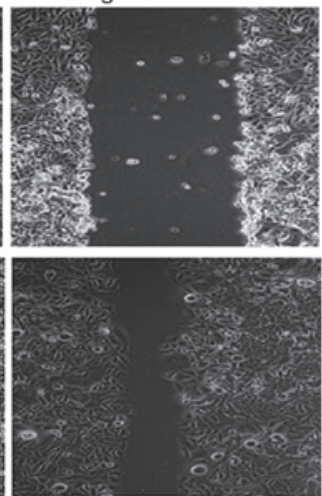

Negative-shRNA

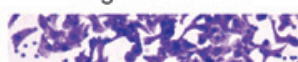

Notch2-shRNA
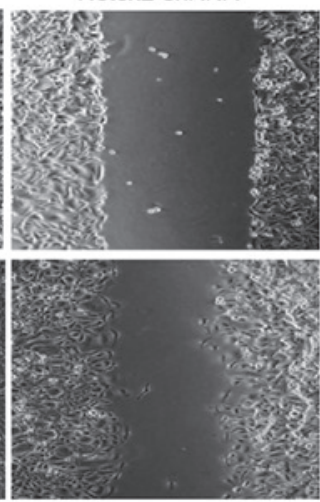

Notch2-shRNA
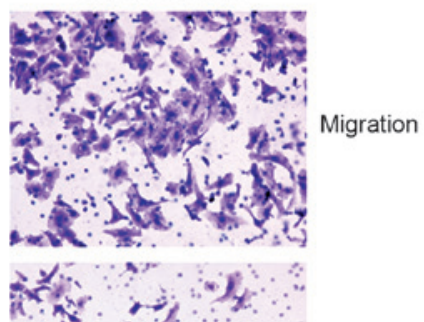

$\rightarrow x^{2}$ i. 1 .

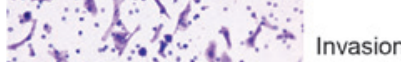

B

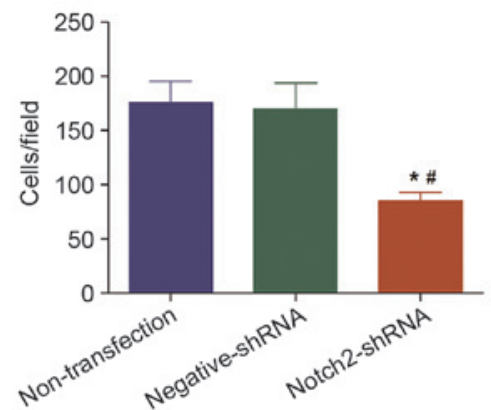

D

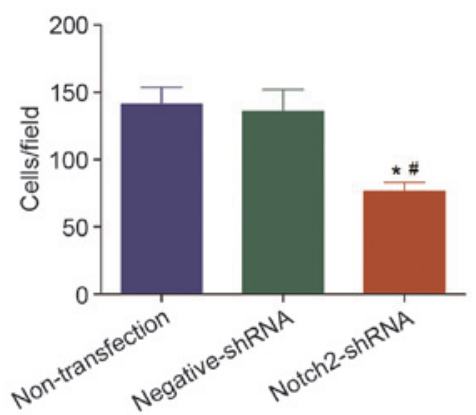

Figure 4. Knockdown of NOTCH2 inhibits the migration and invasion of Hep-2 cells. (A) NOTCH2 shRNA transfected Hep-2 cells exhibited inhibited cellular motility compared with the control cells. (B) The number of cells that migrated through uncoated filters (no Matrigel) represents the migratory ability of Hep-2 cells. (C) Representative images of the Transwell assay without Matrigel (upper panel) or pre-coated with Matrigel (lower panel) following shRNA transfection. (D) The number of cells that were able to pass through filters pre-coated with Matrigel represents the invasive ability of Hep-2 cells. The cell counts are presented as the mean \pm standard deviation of $\geq 5$ randomly selected low-power fields (x200) from three independent experiments. "P<0.05 vs. non-transfected samples, ${ }^{\text {}} \mathrm{P}<0.05$ vs. negative-shRNA samples. shRNA, short hairpin RNA. 
A

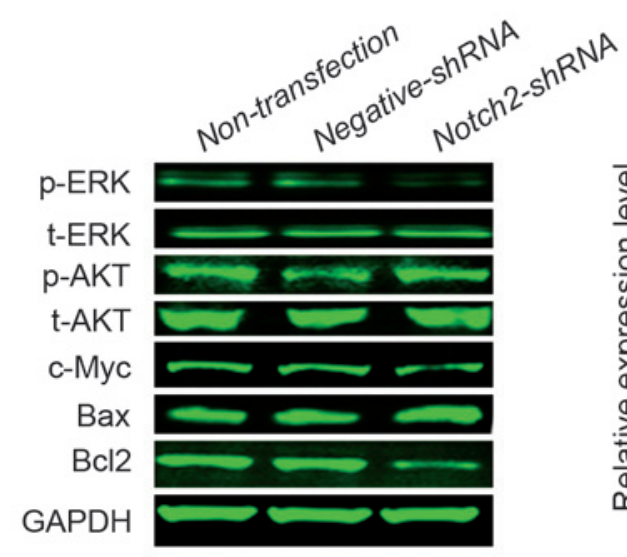

B

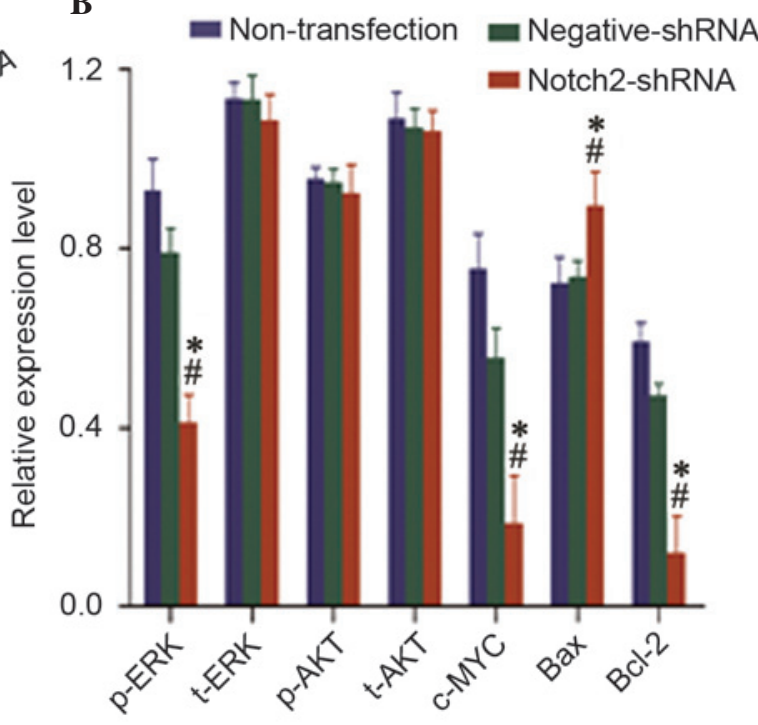

Figure 5. Knockdown of NOTCH2 affects the expression of Notch 2 signaling pathway target genes in Hep-2 cells. (A) Western blot analysis demonstrating that the expression levels of p-ERK, c-Myc and Bcl-2 were downregulated, and the expression of Bax was upregulated. No differences were observed in the expression of t-ERK, p-Akt and t-Akt. (B) Quantification of the protein bands by densitometry. The results of three independent experiments are presented. All the histograms present the GAPDH-normalized mean \pm standard deviation of the band density from the three experiments. ${ }^{*} \mathrm{P}<0.05$ vs. non-transfected cells, ${ }^{~} \mathrm{P}<0.05$ vs. negative shRNA. shRNA, short hairpin RNA; p, phospho; t, total; ERK, mitogen-activated protein kinase; AKT, $v$-akt murine thymoma viral oncogene homolog 1; c-Myc, $v$-myc avian myelocytomatosis viral oncogene homolog; Bax, BCL2-associated X protein; Bcl2, B-cell CLL/lymphoma 2.

respectively $(\mathrm{P}=0.011$ and $\mathrm{P}=0.013$, respectively; Fig. $2 \mathrm{~A})$. Similarly, Notch 2 protein levels were reduced by 82.0 and $80.3 \%$ $(\mathrm{P}=0.011$ and $\mathrm{P}=0.013$, respectively; Fig. 2B). Additionally, no significant difference was observed in the levels of mRNA or protein between the cells that were non-transfected and those transfected with negative-shRNA (P>0.05; Fig. 2).

NOTCH2 knockdown decreases cell proliferation and increases cell apoptosis in Hep-2 cells. The present study investigated the effect of Notch 2 on the cell morphology, proliferation and apoptosis of LSCC. Following NOTCH2 knockdown, the Hep-2 cells underwent morphological changes, consistent with reduced cell numbers in culture (Fig. 3A). Similarly, NOTCH2 knockdown significantly inhibited the growth of the Hep-2 cells compared with negative-shRNA and non-transfected cells, as measured using CCK-8 reagent $(\mathrm{P}=0.028$ and $\mathrm{P}=0.046$, respectively; Fig. $3 \mathrm{~B})$. In addition to the decreased percentage of proliferating cells, the percentage of apoptotic cells were increased, compared with negative-shRNA and non-transfected cells, as measured by flow cytometry (both $\mathrm{P}=0.003$; Fig. $3 \mathrm{C}$ and $\mathrm{D}$ ).

Notch 2 increases cell migration and invasion in vitro. The present study additionally investigated the effect of Notch 2 on the migration and invasion abilities of LSCC. Compared with negative-shRNA and non-transfected Hep-2 cells, knockdown of NOTCH2 mRNA significantly inhibited cell migration $(\mathrm{P}<0.05$; Fig. $4 \mathrm{~A}$ and $\mathrm{B})$ and invasion $(\mathrm{P}<0.05$; Fig. 4C and D). These results support the theory that NOTCH2 is an oncogene that may contribute to the migration and invasion of LSCC.

Notch 2 affects the expression of cell proliferation-and apoptosis-associated proteins. To investigate the effect of Notch pathway proteins on the apoptosis of cancer cells, the expression levels of proteins associated with the Notch pathway were evaluated. Following knockdown of Notch 2 protein expression, the protein expression levels of phospho-mitogen-activated protein kinase 1 (p-ERK) $(\mathrm{P}=0.003$ and $\mathrm{P}=0.016), \mathrm{c}-\mathrm{Myc}(\mathrm{P}=0.011$ and $\mathrm{P}=0.048)$ and $\mathrm{Bcl} 2(\mathrm{P}=0.003$ and $\mathrm{P}=0.012)$ were also downregulated $(\mathrm{P}<0.05)$, whereas the expression level of $\mathrm{Bcl} 2$-associated $\mathrm{X}$ protein $(\mathrm{Bax})$ was upregulated $(\mathrm{P}<0.05)$ compared with negative-shRNA and non-transfected Hep-2 cells (Fig. 5). Notch 2 knockdown demonstrated no effect on the protein expression levels of total-ERK (t-ERK), phospho-v-akt murine thymoma viral oncogene homolog 1 (p-Akt) or total-Akt (t-Akt) ( $\mathrm{P}>0.05$; Fig. 5).

\section{Discussion}

The Notch signaling pathway has been associated with the initiation and development of various types of human cancer, including breast, brain, cervix, lung, colon, head and neck, kidney, bone marrow, lymph nodes and stomach cancer $(5,21-25)$. Therefore, Notch signaling has become an attractive anticancer drug target (26). However, the molecular alterations of the Notch signaling pathways in LSCC are less well defined and the precise mechanisms of Notch 2-mediated tumor proliferation and anti-apoptotic effects remain unclear. The present study aimed to investigate the importance of the Notch 2 signaling pathway in LSCC tissues and Hep-2 laryngeal cancer cells.

The current study demonstrated that Notch 2 expression was upregulated in LSCC tissues compared with tissues from normal vocal polyps. This upregulation was increased further in tissues with lymph node metastasis compared with LSCC tissues without lymph node metastasis, indicating that Notch 2 
may be oncogenic during the tumorigenesis and metastasis of LSCC.

The present study additionally demonstrated that shRNA-mediated downregulation of Notch 2 expression in Hep-2 laryngeal cancer cells inhibited cell proliferation and induced cell apoptosis. It was observed that NOTCH2 mRNA knockdown decreased Hep-2 cell proliferation compared with controls. In addition, the decreased expression of Notch 2 resulted in an increased proportion of apoptotic cells, as measured by flow cytometry. Again, this result suggests that $\mathrm{NOTCH} 2$ is an oncogene that may contribute to the proliferation and evasion of apoptosis in LSCC.

Previous studies have demonstrated that the Notch receptors regulate cell proliferation and apoptosis through multiple downstream pathways $(2,27,28)$. The protein expression of Notch 2, in particular, has been demonstrated to affect downstream signaling pathways in different carcinoma cells $(2,29,30)$. The current study examined the expression levels of the conventional Notch target genes in Hep-2 cells. It was observed that the silencing of Notch 2 resulted in decreased phosphorylation levels of ERK, and decreased expression levels of $\mathrm{c}-\mathrm{Myc}$ and $\mathrm{Bcl} 2$, whereas the expression of the pro-apoptotic protein Bax was upregulated. However, no changes were observed in the expression of t-ERK, p-Akt and t-Akt following NOTCH 2 knockdown. The biological effects of ERK are induced via p-ERK1/2 (31). Zhou et al (32) demonstrated that the Notch inhibitor, DAPT, reduced ERK1/2 activity and decreased the protein expression levels of matrix metalloproteinase (MMP)-2, MMP-9 and vascular endothelial growth factor, resulting in the suppression of hepatocellular carcinoma invasion. Asnaghi et al (33) reported that Notch blockade reduced ERK activity in uveal melanoma cells. They implied that the Notch signaling pathway may modulate ERK activity to regulate cell proliferation and apoptosis. The results of the current study demonstrated that NOTCH 2 mRNA knockdown decreased the phosphorylation level of ERK, which indicates reduced ERK activity. Therefore, the downregulation of Notch 2 inhibited the proliferation of Hep-2 cells by altering the activity and expression of cell proliferation and apoptosis-associated proteins.

Furthermore, the present study demonstrated that NOTCH2 knockdown inhibits the in vitro migration and invasion ability of Hep-2 cells, however, the underlying molecular mechanisms involved remain unclear (34). Further studies are required to precisely determine the importance of Notch 2 signaling pathways in the invasion and lymph node metastasis of LSCC.

In summary, the results of the current study indicate that Notch 2 is highly expressed in LSCC tissues and is associated with increased tumorigenesis and metastasis. Additionally, knockdown of NOTCH2 inhibits cell proliferation, induces apoptosis, and decreases cell migration and invasion of Hep-2 cancer cells in vitro. Therefore, the results of the present study suggest that the Notch 2 signaling pathway is important in the cell growth, apoptosis regulation and metastasis of LSCC. Together, these results suggest that the development of Notch 2 inhibitors to decrease cancer cell growth and metastasis may be a promising therapeutic strategy for the treatment of patients with LSCC.

\section{Acknowledgements}

The present work was supported by the National Natural Science Foundation of China (grant nos. 81172569 and 81372880).

\section{References}

1. Wen PY and Kesari S: Malignant gliomas in adults. N Engl J Med 359: 492-507, 2008.

2. Ramdass B, Maliekal TT, Lakshmi S, Rehman M, Rema P, Nair P, Mukherjee G, Reddy BK, Krishna S and Radhakrishna Pillai M: Coexpression of Notch1 and NF kappaB signaling pathway components in human cervical cancer progression. Gynecol Oncol 104: 352-361, 2007.

3. Liu M, Wu H, Liu T, Li Y, Wang F, Wan H, Li X and Tang H: Regulation of the cell cycle gene, BTG2, by miR-21 in human laryngeal carcinoma. Cell Res 19: 828-837, 2009.

4. Li JL, Sainson RC, Oon CE, Turley H, Leek R, Sheldon H, Bridges E, Shi W, Snell C, Bowden ET, et al: DLL4-Notch signaling mediates tumor resistance to anti-VEGF therapy in vivo. Cancer Res 71: 6073-6083, 2011.

5. Capaccione KM and Pine SR: The Notch signaling pathway as a mediator of tumor survival. Carcinogenesis 34: 1420-1430, 2013.

6. Ntziachristos P, Lim JS, Sage J and Aifantis I: From fly wings to targeted cancer therapies: A centennial for notch signaling. Cancer Cell 25: 318-334, 2014.

7. Bin Hafeez B, Adhami VM, Asim M, Siddiqui IA, Bhat KM, Zhong W, Saleem M, Din M, Setaluri V and Mukhtar H: Targeted knockdown of Notch1 inhibits invasion of human prostate cancer cells concomitant with inhibition of matrix metalloproteinase-9 and urokinase plasminogen activator. Clin Cancer Res 15: 452-459, 2009.

8. Wang Z, Li Y, Banerjee S, Kong D, Ahmad A, Nogueira V, Hay $\mathrm{N}$ and Sarkar FH: Down-regulation of Notch-1 and Jagged-1 inhibits prostate cancer cell growth, migration and invasion, and induces apoptosis via inactivation of Akt, mTOR, and NF-kappaB signaling pathways. J Cell Biochem 109: 726-736, 2010.

9. Yeh TS, Wu CW, Hsu KW, Liao WJ, Yang MC, Li AF, Wang AM, Kuo ML and Chi CW: The activated Notch1 signal pathway is associated with gastric cancer progression through cyclooxygenase-2. Cancer Res 69: 5039-5048, 2009.

10. Chiaramonte R, Basile A, Tassi E, Calzavara E, Cecchinato V, Rossi V, Biondi A and Comi P: A wide role for NOTCH1 signaling in acute leukemia. Cancer Lett 219: 113-120, 2005.

11. Mirandola L, Comi P, Cobos E, Kast WM, Chiriva-Internati M and Chiaramonte R: Notch-ing from T-cell to B-cell lymphoid malignancies. Cancer Lett 308: 1-13, 2011.

12. Weng AP, Ferrando AA, Lee W, Morris JP IV, Silverman LB, Sanchez-Irizarry C, Blacklow SC, Look AT and Aster JC: Activating mutations of NOTCH1 in human T cell acute lymphoblastic leukemia. Science 306: 269-271, 2004.

13. Chiaramonte R: Still puzzling Notch signaling in B-cell malignancies. Leuk Res 30: 1331-1332, 2006.

14. Hubmann R, Schwarzmeier JD, Shehata M, Hilgarth M, Duechler M, Dettke M and Berger R: Notch2 is involved in the overexpression of CD23 in B-cell chronic lymphocytic leukemia. Blood 99: 3742-3747, 2002.

15. Trøen G, Wlodarska I, Warsame A, Hernández Llodrà S, De Wolf-Peeters C and Delabie J: NOTCH2 mutations in marginal zone lymphoma. Haematologica 93: 1107-1109, 2008.

16. Egloff AM and Grandis JR: Molecular pathways: Context-dependent approaches to Notch targeting as cancer therapy. Clin Cancer Res 8: 5188-5195, 2012.

17. Leong KG and Karsan A: Recent insights into the role of Notch signaling in tumorigenesis. Blood 107: 2223-2233, 2006.

18. Yin L, Velazquez OC and Liu ZJ: Notch signaling: Emerging molecular targets for cancer therapy. Biochem Pharmacol 80: 690-701, 2010.

19. Chu D, Li Y, Wang W, Zhao Q, Li J, Lu Y, Li M, Dong G, Zhang H, Xie H and Ji G: High level of Notch1 protein is associated with poor overall survival in colorectal cancer. Ann Surg Oncol 17: 1337-1342, 2010. 
20. Livak KJ and Schmittgen TD: Analysis of relative gene expression data using real-time quantitative PCR and the 2(-Delta Delta C(T)) Method. Methods 25: 402-8, 2001.

21. Wang J, Wang C, Meng Q, Li S, Sun X, Bo Y and Yao W: siRNA targeting Notch 1 decreases glioma stem cell proliferation and tumor growth. Mol Biol Rep 39: 2497-2503, 2012.

22. Ristorcelli E and Lombardo D: Targeting Notch signaling in pancreatic cancer. Expert Opin Ther Targets 14: 541-552, 2010

23. Jundt F, Anagnostopoulos I, Förster R, Mathas S, Stein H and Dörken B: Activated Notch1 signaling promotes tumor cell proliferation and survival in Hodgkin and anaplastic large cell lymphoma. Blood 99: 3398-3403, 2002.

24. Liu ZJ, Xiao M, Balint K, Smalley KS, Brafford P, Qiu R, Pinnix CC, Li X and Herlyn M: Notch1 signaling promotes primary melanoma progression by activating mitogen-activated protein kinase/phosphatidylinositol 3-kinase-Akt pathways and up-regulating N-cadherin expression. Cancer Res 66: 4182-4190, 2006

25. Wang Z, Zhang Y, Li Y, Banerjee S, Liao J and Sarkar FH: Down-regulation of Notch-1 contributes to cell growth inhibition and apoptosis in pancreatic cancer cells. Mol Cancer Ther 5: 483-493, 2006.

26. Espinoza I and Miele L: Notch inhibitors for cancer treatment. Pharmacol Ther 139: 95-110, 2013.

27. Jin R, Nakada M, Teng L, Furuta T, Sabit H, Hayashi Y, Demuth T, Hirao A, Sato H, Zhao G and Hamada J: Combination therapy using Notch and Akt inhibitors is effective for suppressing invasion but not proliferation in glioma cells. Neurosci Lett 534: 316-321, 2013.
28. Sivasankaran B, Degen M, Ghaffari A, Hegi ME, Hamou F, Ionescu MC, Zweifel C, Tolnay M, Wasner M, Mergenthaler S, et al: Tenascin $\mathrm{C}$ is a novel RBPJkappa induced target gene for Notch signaling in gliomas. Cancer Res 69: 458-465, 2009.

29. Luo DH, Zhou Q, Hu SK, Xia YQ, Xu CC, Lin TS, Pan YT, Wu JS and Jin R: Differential expression of Notch1 intracellular domain and 21 proteins, and their clinical significance in gastric cancer. Oncol Lett 7: 471-478, 2014.

30. Ramakrishnan V, Ansell S, Haug J, Grote D, Kimlinger T, Stenson M, Timm M, Wellik L, Halling T, Rajkumar SV and Kumar S: MRK003, a $\gamma$-secretase inhibitor exhibits promising in vitro pre-clinical activity in multiple myeloma and non-Hodgkin's lymphoma. Leukemia 26: 340-348, 2012.

31. Widmann C, Gibson S,Jarpe MB and Johnson GL: Mitogen-activated protein kinase: Conservation of a three-kinase module from yeast to human. Physiol Rev 79: 143-180, 1999.

32. Zhou L, Wang DS, Li QJ, Sun W, Zhang Y and Dou KF: Downregulation of the Notch signaling pathway inhibits hepatocellular carcinoma cell invasion by inactivation of matrix metalloproteinase- 2 and -9 and vascular endothelial growth factor. Oncology Reports 28: 874-882, 2012.

33. Asnaghi L, Ebrahimi KB, Schreck KC, Bar EE, Coonfield ML, Bell WR, Handa J, Merbs SL, Harbour JW and Eberhart CG: Notch signaling promotes growth and invasion in uveal melanoma . Clin Cancer Res 18: 654-665, 2012

34. Pickering CR, Zhou JH, Lee JJ, Drummond JA, Peng SA, Saade RE, Tsai KY, Curry JL, Tetzlaff MT, Lai SY, et al: Mutational landscape of aggressive cutaneous squamous cell carcinoma. Clin Cancer Res 20: 6582-6592, 2014 